\title{
Toward a Theory of High-Temperature Superconductivity in the Antiferromagnetically
} Correlated Cuprate Oxides

\author{
P. Monthoux, ${ }^{(1)}$ A. V. Balatsky, ${ }^{(1),(2),(a)}$ and D. Pines ${ }^{(1)}$ \\ (1)Department of Physics, University of Illinois at Urbana-Champaign, 1110 West Green Street, Urbana, Illinois 61801 \\ ${ }^{(2)}$ Landau Institute for Theoretical Physics, Moscow, U.S.S.R.
} (Received 17 July 1991)

\begin{abstract}
We show that the retarded interaction between quasiparticles on a $2 \mathrm{D}$ square lattice induced by the exchange of antiferromagnetic paramagnons leads uniquely to a transition to a superconducting state with $d_{x^{2}-y^{2}}$ symmetry. With a spin-excitation spectrum and a quasiparticle-paramagnon coupling determined by fits to normal-state experiments, we obtain high transition temperatures and energy-gap behaviors comparable to those measured for $\mathrm{YBa}_{2} \mathrm{Cu}_{3} \mathrm{O}_{7}, \mathrm{YBa}_{2} \mathrm{Cu}_{3} \mathrm{O}_{6.63}$, and $\mathrm{La}_{1.85} \mathrm{Sr}_{0.15} \mathrm{CuO}_{4}$.
\end{abstract}

PACS numbers: 74.65.+n, 05.30.-d, 72.20.-i, 75.10.Jm

During the five years which have elapsed since the discovery of high-temperature superconductivity in cuprate oxide compounds [1], it has become increasingly clear that the normal-state properties of the cuprate oxides are qualitatively different from those of the "lowtemperature superconductors" while their superconducting properties can be understood qualitatively using a BCS approach [2]. Attention has therefore focused on understanding the physical origin of the novel properties of the normal state in the hope and expectation that these would provide an essential clue to the appearance of high-temperature superconductivity. It has been argued that it is the strong antiferromagnetic correlations of the nearly localized $\mathrm{Cu}^{2+} d$ orbitals which are chiefly responsible for the unusual properties of the normal state [3]. In this Letter we examine the possibility that these are also responsible for their high-temperature superconductivity.

Well-established NMR experiments [4] in conjunction with a phenomenological one-component theory [5] demonstrate that for the normal state of the cuprate oxide superconductors the imaginary part of the planar spin-spin correlation function $\chi^{\prime \prime}(\mathbf{q}, \omega)$ is sharply peaked at $\mathbf{q}=( \pm \pi / a, \pm \pi / a)$ in the Brillouin zone (BZ), and that the dominant magnetic excitations are temperaturedependent low-frequency antiferromagnetic paramagnons with a characteristic energy $\omega_{\mathrm{SF}}$ that is always less than $k_{B} T[5,6]$. Quasiparticle-paramagnon scattering has been shown to give rise to a resistivity which is linear in temperature [7], to the anomalous low-frequency optical properties which are seen experimentally [8], and to an imaginary part of the quasiparticle self-energy which is proportional to $\omega$ at low frequencies [9]. We present here the results of calculations on a $2 \mathrm{D}$ square lattice which show that the induced quasiparticle interaction produced by these experimentally determined magnetic excitations leads to a transition, at comparatively high temperatures, to a superconducting state with a $d$-wave gap $\Delta$ $\propto \cos \left(k_{x} a\right)-\cos \left(k_{y} a\right)$, whose symmetry agrees with previous calculations of spin-fluctuation-induced superconductivity for heavy-electron systems and the cuprate oxides [10]. Although our numerical solution for the transition temperature $T_{c}$ is well approximated by a BCS-like formula, we find that in the vicinity of $T_{c}$ the gap develops more rapidly than in weak-coupling BCS theory, while the maximum-gap ratio, $\Delta_{\max }(0) / k_{B} T_{c}$, ranges from 2.9 to 4.3 , depending on the high- $T_{c}$ compound $\left(\mathrm{YBa}_{2} \mathrm{Cu}_{3} \mathrm{O}_{7}, \mathrm{YBa}_{2} \mathrm{Cu}_{3} \mathrm{O}_{6.63}\right.$, or $\left.\mathrm{La}_{1.85} \mathrm{Sr}_{0.15} \mathrm{CuO}_{4}\right)$ we consider. The values of the quasiparticle-paramagnon coupling deduced from the measured values of $T_{c}$ provide a reasonable quantitative account of the above-mentioned anomalous normal-state properties.

We follow Anderson [11] and use a one-band description of the planar excitations of a 2D square lattice; however, instead of introducing spinons and holons we assume the planar excitations form an almost antiferromagnetic Fermi liquid made up of quasiparticles coupled to spin fluctuations by

$$
\mathscr{H}_{\text {int }}=\frac{1}{\Omega} \sum_{\mathbf{q}} \bar{g}(\mathbf{q}) \mathbf{s}(\mathbf{q}) \cdot \mathbf{S}(-\mathbf{q}),
$$

where $\mathbf{s}(\mathbf{q})=\frac{1}{2} \sum_{\alpha, \beta, \mathbf{k}} \psi_{\mathbf{k}+\mathbf{q}, \alpha}^{\dagger} \sigma_{\alpha \beta} \psi_{\mathbf{k}, \beta} . \quad \mathbf{S}$ is the spin-fluctuation operator whose correlation function is modeled by

$$
\chi(\mathbf{q}, \omega)=\frac{\chi_{\mathbf{Q}}}{1+\xi^{2}(\mathbf{q}-\mathbf{Q})^{2}-i \omega / \omega_{\mathbf{S F}}}, \quad q_{x}>0, \quad q_{y}>0,
$$

where $\chi_{\mathrm{Q}}$ is the static spin susceptibility at wave vector $\mathbf{Q}=(\pi / a, \pi / a)$. In the normal state, $\chi_{\mathbf{Q}} \equiv \chi_{0}(\xi / a)^{2} \beta^{1 / 2}$, where $\chi_{0}$ is the experimentally measured long-wavelength spin susceptibility which is in general temperature dependent, $\xi$ is the temperature-dependent antiferromagnetic correlation length, and $\beta \approx \pi^{2}$. The paramagnon energy is given by

$$
\omega_{\mathrm{SF}}=\frac{\Gamma}{\beta^{1 / 2} \pi(\xi / a)^{2}},
$$

where $\Gamma \approx 0.4 \mathrm{eV}$ plays the role of a magnetic Fermi energy. The fits to NMR experiments [5-7] yield the values shown in Table I for $\xi\left(T_{c}\right)$ and $\omega_{\mathrm{SF}}\left(T_{c}\right)$. Since those experiments also indicate that the antiferromagnetic correlations become frozen in the superconducting state, the parameters $\Gamma, \xi, \omega_{\mathrm{SF}}$, and $\chi_{\mathbf{Q}}$ are taken to be constants below $T_{c}$.

In the case of $\mathrm{YBa}_{2} \mathrm{Cu}_{3} \mathrm{O}_{7}$, the spin susceptibility $\chi_{0}$ which enters into Eq. (2) is independent of temperature, 
TABLE I. Input spin excitation and calculated pairing parameters for cuprate superconductors.

\begin{tabular}{lccc}
\hline \hline & & Superconductor & \\
& $\mathrm{YBa}_{2} \mathrm{Cu}_{3} \mathrm{O}_{7}$ & $\mathrm{YBa}_{2} \mathrm{Cu}_{3} \mathrm{O}_{6.63}$ & $\mathrm{La}_{1.85} \mathrm{Sr}_{0.15} \mathrm{CuO}_{4}$ \\
\hline$\chi_{0}\left(T_{c}\right)$ (states/eV) & 2.62 & 0.75 & 1.20 \\
$\xi\left(T_{c}\right) / a$ & 2.5 & 4.1 & 6.5 \\
$\omega_{\mathrm{SF}}\left(T_{c}\right)(\mathrm{K})$ & 90 & 35 & 10 \\
$\lambda\left(T_{c}\right)$ & 0.477 & 0.373 & 0.331 \\
$\Delta_{\text {max }}(0) / k_{B} T_{c}$ & 2.9 & 3.4 & 4.3 \\
$x^{\mathrm{b}}$ & 0.45 & 0.33 & 0.30 \\
\hline \hline
\end{tabular}

${ }^{"} T_{c} \propto e-1 / \lambda\left(T_{c}\right)$.

${ }^{\mathrm{b}} \Delta_{\operatorname{mix}}(T) /\left.\Delta_{\max }(0)\right|_{T \cdot T_{c}} \propto\left(T / T_{c}-1\right)^{x}$.

and, with a coupling constant $\bar{g}(\mathbf{q})$ which is temperature and wave vector independent, one obtains a normal-state resistivity which is linear in $T$. For $\mathrm{YBa}_{2} \mathrm{Cu}_{3} \mathrm{O}_{6.63}$ and $\mathrm{La}_{1.85} \mathrm{Sr}_{0.15} \mathrm{CuO}_{4}$ the uniform spin susceptibility is markedly temperature dependent. We find that in order to obtain a linear resistivity, $\bar{g}(r)$ must be temperature dependent. A convenient form for large $r$ is $\bar{g}(r)$ $\propto(-1)^{\left(n_{x}+n_{y}\right)} \exp [-r / l(T)] / r, \quad \mathbf{r}=\left(n_{x} a, n_{y} a\right), \quad$ where $l(T)$ is chosen in such a way that the product of $\chi_{0}(T)$ and a temperature-dependent effective coupling,

$$
\bar{g}_{\mathrm{eff}}^{2}(T) \stackrel{\text { def }}{\equiv} \frac{\sum_{\mathbf{q}} \bar{g}^{2}(\mathbf{q}) \chi(\mathbf{q}, \omega \rightarrow 0)}{\sum_{\mathbf{q}} \chi(\mathbf{q}, \omega \rightarrow 0)},
$$

is nearly independent of $T$. We find that $l(T)$ varies from $\sim 3(\sim 2.1)$ lattice spacings at $T=60 \mathrm{~K}$ to $\sim 1.5$ ( 1.7) lattice spacings at $T=250 \mathrm{~K}$ for the $\mathrm{YBa}_{2} \mathrm{Cu}_{3}$ $\mathrm{O}_{6.63}\left(\mathrm{La}_{1.85} \mathrm{Sr}_{0.15} \mathrm{CuO}_{4}\right)$ compound.

In the weak-coupling limit, we write the linearized gap equation determining the critical temperature $T_{c}$ as

$$
\begin{array}{r}
\Delta(\mathbf{k})=\left(\begin{array}{r}
-g_{\mathrm{eff}}^{2}(T) \\
\frac{1}{3} g_{\mathrm{eff}}^{2}(T)
\end{array}\right) \int_{0}^{\pi / a} \frac{a d k_{x}^{\prime}}{2 \pi} \int_{0}^{k_{x}^{\prime}} \frac{a d k_{y}^{\prime}}{2 \pi} \sum_{i=1}^{8}\left[\operatorname{Re} \chi\left(\mathbf{k}-\Phi_{i} \mathbf{k}^{\prime}, \epsilon_{\mathbf{k}^{\prime}}-\mu\right) \frac{\tanh \left[\left(\epsilon_{\mathbf{k}^{\prime}}-\mu\right) / 2 k_{B} T\right]}{\epsilon_{\mathbf{k}^{\prime}}-\mu}\right. \\
+2 \int_{0}^{\infty} \frac{d \omega}{\pi} \operatorname{coth}\left(\frac{\omega}{2 k_{B} T}\right) \operatorname{Im} \chi\left(\mathbf{k}-\Phi_{i} \mathbf{k}^{\prime}, \omega\right) \\
\left.\times \frac{\left(\epsilon_{\mathbf{k}^{\prime}}-\mu\right)^{2}-\omega^{2}+\delta^{2}}{\left[\left(\epsilon_{\mathbf{k}^{\prime}}-\mu\right)^{2}-\omega^{2}+\delta^{2}\right]^{2}+4 \delta^{2} \omega^{2}}\right] \Delta\left(\Phi_{i} \mathbf{k}^{\prime}\right),
\end{array}
$$

where the $\Phi_{i}$ are the group transformations which map any wave vector in the BZ onto the first octant. The upper coupling factor, $-g_{\mathrm{efr}}^{2}(T)$, is for singlet (repulsive interaction) and the lower one for triplet pairing (attractive interaction). We find that even for small values of the lifetime parameter $\delta$ the second term in the interaction may be neglected compared to the first, while the frequency dependence of the susceptibility cuts off the interaction when $\epsilon_{\mathbf{k}^{\prime}}-\mu$ $\geq \omega_{\mathrm{SF}}\left(\xi^{2} / a^{2}\right) \equiv \Gamma / \pi^{2}$. A tight-binding form of the quasiparticle spectrum $\epsilon_{\mathbf{k}}=-2 t\left[\cos \left(k_{x} a\right)+\cos \left(k_{y} a\right)\right]$ is assumed and the bandwidth is taken as $2 \mathrm{eV}$. The effective coupling constant is $g_{\mathrm{eff}}^{2}(T)=\frac{3}{8} \bar{g}_{\mathrm{eff}}^{2}(T)$.

We find that our interaction leads to a solution of the gap equation only for a $d_{x^{2}-y^{2}}$ pairing state which can be written as $\Delta(\mathbf{k})=\Delta_{0}(\mathbf{k})\left[\cos \left(k_{x} a\right)-\cos \left(k_{y} a\right)\right]$. Because of the structure of the quasiparticle spectrum and the gap $\Delta(\mathbf{k})$, it is convenient to write the integral equation (5) for $\Delta_{0}(\mathbf{k})$ in terms of the new variables $\epsilon$ and $\theta$ :

$$
\begin{aligned}
& k_{x} a=\arccos [-\epsilon-(1-|\epsilon|) \cos (\theta)], \quad k_{y} a=\arccos [-\epsilon+(1-|\epsilon|) \cos (\theta)], \\
& -1 \leq \epsilon \leq 1, \quad 0 \leq \theta \leq \pi / 2 .
\end{aligned}
$$

We neglect the $\theta$ dependence of $\Delta_{0}$, and write Eq. (5) as a one-dimensional integral equation in the variable $\epsilon$ :

$$
\bar{\Delta}(\epsilon)=\frac{g_{\mathrm{eff}}^{2}(T)}{B \pi^{2}} \int_{-1}^{+1} d \epsilon^{\prime} V\left(\epsilon, \epsilon^{\prime}\right) \frac{\tanh \left[B\left(\epsilon^{\prime}-\mu^{\prime}\right) / 4 k_{B} T\right]}{\epsilon^{\prime}-\mu^{\prime}} \bar{\Delta}\left(\epsilon^{\prime}\right),
$$

where

$$
V\left(\epsilon, \epsilon^{\prime}\right) \equiv-\int_{0}^{\pi / 2} d \theta \int_{0}^{\pi / 2} d \theta^{\prime} \frac{\cos \left(\theta^{\prime}\right) \sum_{i=1}^{8}(-1)^{\Phi_{i}} \operatorname{Re} \chi\left(\mathbf{k}(\epsilon, \theta)-\Phi_{i} \mathbf{k}^{\prime}\left(\epsilon^{\prime}, \theta^{\prime}\right), \frac{1}{2} B\left(\epsilon^{\prime}-\mu^{\prime}\right)\right)}{\left[\left(1+\left|\epsilon^{\prime}\right|\right)^{2}-\left(1-\left|\epsilon^{\prime}\right|\right)^{2} \cos ^{2}\left(\theta^{\prime}\right)\right]^{1 / 2}}
$$

and $\bar{\Delta}(\epsilon) \equiv \Delta_{0}(\epsilon)(1-|\epsilon|)$. ( $(-1)^{\Phi_{i}}$ denotes the parity of the gap under the transformation $\Phi_{i}, B$ is the bandwidth, and $\mu \equiv \frac{1}{2} B \mu^{\prime}$. The above approximation amounts to expanding $\Delta_{0}$ in powers of $\cos \left(k_{x} a\right)+\cos \left(k_{y} a\right)$ and solving Eq. (5) exactly in that subspace. Because of the considerable structure of the effective interaction, a very fine mesh in the $\epsilon$ variable is needed. Since the solution of the ensuing 164 coupled equations is computationally expensive, we have not ex- 
plored various chemical potentials: $\mu$ was set to $0.25 \mathrm{eV}$ La representative value, corresponding to $N(0)=0.568$ $\mathrm{eV}^{-1}$ ] for the three compounds and its temperature dependence was neglected throughout. We obtain an identical result with $\mu \rightarrow-\mu$ provided we make the substitution $\epsilon \rightarrow-\epsilon$. The temperature dependence of the gap can be calculated via the equation obtained from (5) by changing $\epsilon_{\mathbf{k}}-\mu$ everywhere to $E_{\mathbf{k}} \equiv\left[\left(\epsilon_{\mathbf{k}}\right.\right.$ $\left.-\mu)^{2}+\Delta^{2}(\mathbf{k})\right]^{1 / 2}$.

Our principal results are displayed in Figs. 1 and 2 and Table I, and in the following we comment on them briefly.

(i) As shown in Fig. 1, our numerical solution for $T_{c}$ is well approximated by

$$
\begin{aligned}
T_{c} & =\alpha \hbar \omega_{\mathrm{SF}}\left(T_{c}\right) \frac{\xi^{2}\left(T_{c}\right)}{a^{2}} \exp \left(-\frac{1}{\lambda\left(T_{c}\right)}\right) \\
& \equiv \alpha \frac{\Gamma\left(T_{c}\right)}{\pi^{2}} \exp \left(-\frac{1}{\lambda\left(T_{c}\right)}\right),
\end{aligned}
$$

where the dimensionless effective coupling constant $\lambda\left(T_{c}\right)=\eta g_{\mathrm{eff}}^{2}\left(T_{c}\right) \chi_{0}\left(T_{c}\right) N(0)$ varies from 0.48 to 0.33 depending on the compound, and $\alpha$ and $\eta$ are material constants of order unity.

(ii) Even though the coupling is intermediate to weak, below $T_{\text {c }}$ the energy gap opens up very rapidly, reaching a maximum magnitude large compared to the weakcoupling BCS result, in good qualitative agreement with experiment [2].

(iii) Stronger antiferromagnetic correlations are accompanied by lower values of $T_{c}$ and larger values of the gap ratio $\Delta_{\max }(0) / k_{B} T_{c}$.

(iv) With a physically reasonable Fermi-liquid correc-

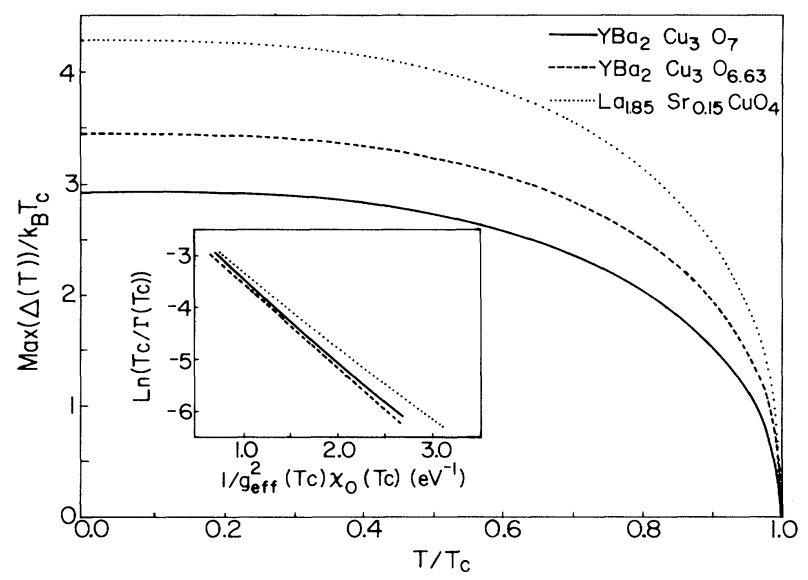

FIG. 1. The temperature dependence of the maximum value of the energy gap for the three high- $T_{c}$ compounds $\mathrm{YBa}_{2} \mathrm{Cu}_{3} \mathrm{O}_{7}$ $\left(T_{c}=95 \mathrm{~K}\right), \mathrm{YBa}_{2} \mathrm{Cu}_{3} \mathrm{O}_{6.63}\left(T_{c}=60 \mathrm{~K}\right)$, and $\mathrm{La}_{1.85} \mathrm{Sr}_{0.15} \mathrm{CuO}_{4}$ $\left(T_{c}=40 \mathrm{~K}\right)$. Inset: A plot of the relationship $T_{c}=\alpha\left[\Gamma\left(T_{c}\right) /\right.$ $\left.\pi^{2}\right] \exp \left[-1 / \eta g_{\text {err }}^{2}\left(T_{c}\right) \chi_{0}\left(T_{c}\right) N(0)\right] . \quad \alpha$ and $\eta$ are material-dependent constants which are found to be $\alpha=1.66, \eta=1.07$ for $\mathrm{YBa}_{2} \mathrm{Cu}_{3} \mathrm{O}_{7} ; \alpha=1.49, \eta=1.07$ for $\mathrm{YBa}_{2} \mathrm{Cu}_{3} \mathrm{O}_{6.63}$; and $\alpha=1.51$, $\eta=1.21$ for $\mathrm{La}_{1.85} \mathrm{Sr}_{0.15} \mathrm{CuO}_{4}$. tion [12], the temperature dependence of our calculated planar spin susceptibility agrees with experiment [13], while the coupling constants, $g_{\text {eff }}^{2}\left(T_{c}\right)$, inferred from $\lambda$ appear to be in the range required to explain the anomalous resistivity and optical properties of the normal state.

In our theory, the high-temperature superconductivity and the unconventional pairing state result from the strong antiferromagnetic correlations responsible for the structure of the effective interaction. The unusual gap behavior results, at least in part, from a nonlinear relation between $\Delta$ and $V\left(\epsilon, \epsilon^{\prime}\right)$ in the superconducting state, produced by the frequency dependence of the susceptibility. Our high values of $T_{c}$ reflect, in part, the fact that apart from an angular average approximation, we have solved the full gap equation (7).

Direct experimental evidence for unconventional pairing in $\mathrm{YBa}_{2} \mathrm{Cu}_{3} \mathrm{O}_{7}$ has been obtained in NMR experiments $[14,15]$ in the superconducting state. The measured temperature-dependent anisotropy of the relaxation rates of the ${ }^{17} \mathrm{O}$ and ${ }^{63} \mathrm{Cu}$ nuclei, for field orientations in the plane and perpendicular to it, appears to rule out conventional $s$-wave pairing, but is qualitatively consistent with the results of $d$-wave pairing calculations [16]. The failure thus far to observe nodes in the gap in experiments on the penetration depth of $\mathrm{YBa}_{2} \mathrm{Cu}_{3} \mathrm{O}_{7}$ may reflect the influence of both impurity scattering and lifetime effects or that the particular geometry of the Fermi surface is such that there is no appreciable contribution to the normal-state density from the nodes. To the extent that lifetime effects are independent of the retardation effects considered here, we would expect that including these would lower the transition temperature, and lead to a still more rapid opening up of the gap near $T_{c}$ and to a still

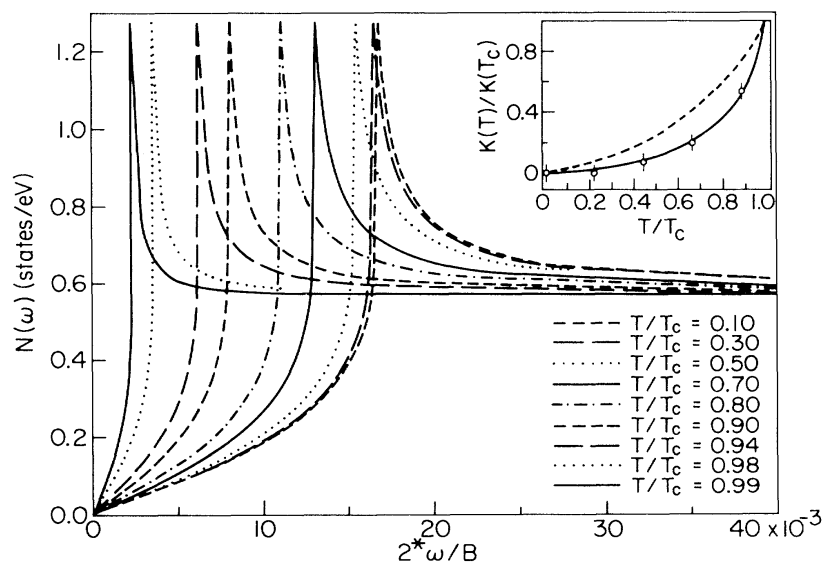

FIG. 2. The density of states for the $d$-wave gap $\Delta^{B_{1}}(\mathbf{k})$ as a function of the variable $2 \omega / B$, where $B=2 \mathrm{eV}$ is the bandwidth, at various values of $T / T_{c}$ for $\mathrm{YBa}_{2} \mathrm{Cu}_{3} \mathrm{O}_{7}$. Note the linear behavior in frequency for small $\omega$ and the logarithmic divergence in two dimensions. Inset: The Knight shift in the superconducting state. The dashed line is the bare value, and the solid line with Fermi-liquid corrections and $F_{0}^{a}=-0.6$. The circles are the experimental values of Barrett et al. [13]. 
larger gap ratio at $T=0$ [17].

Schrieffer and co-workers [18] share our view that antiferromagnetic correlations play a key role in determining the superconducting transition, but argue that the coupling between quasiparticles and spin fluctuations is so strong that the normal-state excitations are solitons (spin bags); they further focus on the second-order spinfluctuation exchange to obtain $s$-wave superconductivity, rather than utilizing the first-order term to obtain $d$-wave pairing.

In our model, the results of experiments on the normal state are used to fix the fundamental quantities which enter the spin-excitation spectrum and its coupling to quasiparticles. We have chosen to use the low-frequency form of $\chi(\mathbf{q}, \omega)$, which fits NMR, because as yet neutron-scattering experiments have not produced a consensus on the behavior of $\chi(\mathbf{q}, \omega)$ in the range $1-50 \mathrm{meV}$. We are encouraged that the results we obtain agree qualitatively in many ways with experiments on the superconducting state. The present calculations, however, represent only a first step toward the development of a consistent theory of superconductivity in the cuprate oxides. For example, before a quantitative comparison with experiment on the transition temperature, gap properties, etc., can be made, it is important to incorporate lifetime effects and to carry out the self-consistent calculation of the chemical potential and its variation with doping which is needed as well for a quantitative account of normal-state properties. While we find it is possible to obtain large energy gaps in a weak-coupling approximation, the role of strong-coupling corrections needs to be explored.

The problem of the nature of the superconducting state in two dimensions in the presence of antiferromagnetic correlations that we have studied here is interesting in its own right. However, the superconductivity in high- $T_{c}$ compounds is three dimensional. Interplanar coupling in some form will establish the true 3D coherence of the order parameter. Since the interplanar coupling is weak, we believe that the in-plane symmetry of the gap will remain $d_{x^{2}-y^{2}}$.

We would like to thank E. Abrahams, J. Bardeen, K. Bedell, J.-P. Lu, A. Millis, H. Monien, J. R. Schrieffer, C. P. Slichter, and D. Thelen for stimulating discussions on these and related topics, and E. Abrahams and $\mathrm{A}$. Millis for numerous helpful suggestions during the preparation of this manuscript. One of us (D.P.) wishes to thank the Santa Fe Institute for its hospitality and the Robert Maxwell Professorship for its support during the preparation of this manuscript. One of us (P.M.) thanks the University of Illinois for its support via a fellowship. This work was supported by NSF Grants No. DMR 88-
17613 and No. DMR 89-20538, and by the National Science Foundation (No. DMR 88-09854) through the Science and Technology Center for Superconductivity.

(a) Present address: Center for Materials Science, Los Alamos National Laboratory, Los Alamos, NM 87545.

[1] J. G. Bednorz and K. A. Mueller, Z. Phys. B 64, 189 (1986).

[2] High Temperature Superconductivity, edited by K. S. Bedell, D. Coffey, D. E. Meltzer, D. Pines, and J. R. Schrieffer (Addison-Wesley, New York, 1990).

[3] D. Pines, in Ref. [2], pp. 392-396.

[4] P. C. Hammel et al., Phys. Rev. Lett. 63, 1992 (1989); S. E. Barrett et al., Phys. Rev. B 41, 6283 (1990); T. Imai et al., J. Phys. Soc. Jpn. 57, 1771 (1988); R. E. Walstedt et al., Phys. Rev. B 38, 9299 (1988).

[5] A. J. Millis, H. Monien, and D. Pines, Phys. Rev. B 42, 167 (1990).

[6] H. Monien, M. Takigawa, and D. Pines, Phys. Rev. B 43, 258 (1991); H. Monien, P. Monthoux, and D. Pines, Phys. Rev. B 43, 275 (1991).

[7] Monien, Monthoux, and Pines (Ref. [6]); T. Moriya, Y. Takahashi, and K. Ueda, J. Phys. Soc. Jpn. 59, 2095 (1990).

[8] B. Arfi (to be published); T. Moriya and Y. Takahashi (to be published).

[9] P. Monthoux (unpublished); C. M. Varma et al., Phys. Rev. Lett. 63, 1996 (1989).

[10] V. J. Emery, J. Phys. (Paris), Colloq. 44, C3-977 (1983); Synth. Met. 13, 21 (1986); D. J. Scalapino, E. Loh, and J. Hirsch, Phys. Rev. B 34, 8190 (1986); J. E. Hirsch, Phys. Rev. Lett. 54, 1317 (1985); K. Miyake, S. Schmitt-Rink, and C. M. Varma, Phys. Rev. B 34, 6654 (1986); P. A. Lee and N. Read, Phys. Rev. Lett. 58, 2691 (1987); D. J. Scalapino, in MRS Symposia Proceedings Vol. EA-II (Materials Research Society, Pittsburgh, 1987), p. 35; N. E. Bickers, D. J. Scalapino, and R. T. Scalettar, Int. J. Mod. Phys. B 1, 687 (1987).

[11] P. W. Anderson, Science 235, 1196 (1987).

[12] A. J. Leggett, Rev. Mod. Phys. 47, 331 (1975).

[13] S. E. Barrett et al., Phys. Rev. B 41, 6283 (1990).

[14] S. E. Barrett et al., Phys. Rev. Lett. 66, 108 (1991); J. A. Martindale et al. (unpublished).

[15] M. Takigawa, J. L. Smith, and W. L. Hults, Los Alamos Report No. LA-UR-91-1228, 1991 (to be published).

[16] N. Bulut and D. J. Scalapino, University of California, Santa Barbara, Report No. UCSBTH-91-05, 1991 (to be published).

[17] A. Millis, S. Sachdev, and C. M. Varma, Phys. Rev. B 37, 4975 (1988); T. Koyama and M. Tachiki, Phys. Rev. B 39, 2279 (1989); Y. Kuroda and C. M. Varma, Phys. Rev. B 42, 8619 (1990).

[18] J. R. Schrieffer, X.-G. Wen, and S. C. Zhang, Phys. Rev. B 39, 11663 (1989); A. Kampf and J. R. Schrieffer, Phys. Rev. B 41, 6399 (1990); 42, 7967 (1990). 